\title{
Localized time accurate sampling of nonequilibrium and unsteady hypersonic flows: methods and horizons
}

\author{
Richard Miles ${ }^{1,2,3}$ (D) Arthur Dogariu ${ }^{2,4} \cdot$ Laura Dogariu $^{4}$
}

Received: 11 February 2021 / Revised: 12 October 2021 / Accepted: 13 October 2021 / Published online: 22 November 2021

(c) The Author(s) 2021

\begin{abstract}
Modern "non-intrusive" optical methods are providing revolutionary capabilities for diagnostics of hypersonic flow fields. They generate accurate information on the performance of ground test facilities and provide local time accurate measurements of near-wall and off-body flow fields surrounding hypersonic test articles. They can follow the true molecular motion of the flow and detect nonequilibrium states and gas mixtures. They can be used to capture a wide range of turbulent scales and can produce highly accurate velocity, temperature and density measurements as well as time-frozen images that provide intuitive understanding of flow phenomena. Recent review articles address many of these methods and their applications. The methods highlighted in this review are those that have been enabled or greatly improved by new, versatile laser systems, particularly including $\mathrm{kHz}$ rate femtosecond lasers and $\mathrm{MHz}$ rate pulse burst lasers. Although these methods can be applied to combusting environments, the focus of this review is on external high Mach number flows surrounding test articles and wind tunnel core flow properties. The high repetition rates enable rapid time evolving flows to be analyzed and enable the collection of large data sets necessary for statistical analysis. Future capabilities based on the use of atomic vapor filters and on frequency tunable, injection locked MHz rate lasers are promising.
\end{abstract}

\section{Introduction}

As maneuverable air platforms move up in speed from subsonic through supersonic to hypersonic, satisfactory safety, reliability and performance become increasingly difficult to achieve. The trade-off between performance and reliability grows ever more critical, with the loss of a few percent in performance potentially leading to a lost mission capability. The platform design task is made even more challenging by the high cost of flight tests and the constraints of ground testing capabilities. In addition, for flight tests diagnostics are sparse and maneuvers are severely restricted and often cannot fully simulate mission requirements. To overcome these limitations, high fidelity and validated computational models

Richard Miles

rmiles@tamu.edu

1 Department of Aerospace Engineering, Texas A and M University, College Station, USA

2 Department of Mechanical and Aerospace Engineering, Princeton University, Princeton, USA

3 Plasma TEC., Inc., Princeton, USA

4 Speckodyne, Corp., Princeton, USA are needed. These models must incorporate the true physics of the air flow and must be able to predict performance, including laminar to turbulent transition, nonequilibrium phenomena, dissociation, ionization, unsteadiness, shock interactions and separation as well as perturbations caused by ablation, surface degradation and structural coupling. Air breathing platforms add another level of complexity for modeling, including air fuel mixing, flame holding, ignition, combustion efficiency, unstart, sensitivity to maneuvers and thrust generation.

The challenge to the diagnostic community is twofold (1) to capture the relevant physics with sufficient fidelity to inform the modeling community and (2) to provide methods for validating model predictions in relevant environments. In both cases, the data need to be captured with temporal and spatial resolutions that enable detailed processes to be followed and understood. Other diagnostic challenges include the development of sensors to provide feedback control for flight operations and for safety monitoring. For these phenomena to be properly measured, sampling must occur at rates ranging from kiloHertz to megaHertz, often with resolution better than one millimeter.

Laser-based methods for the measurement of hypersonic flows date back more than 40 years (Zimmermann and Miles 
1980) and have been summarized in books (Smits and Lim 2012; Kohse-Hoeinghaus and Jeffries 2002; Hanson et al.. 2016; Eckbreth 1996) and review articles (Miles and Lempert 1997; Miles 2015; Ehn et al.. 2017; De Cesare et al. 2020,). Of particular relevance for the application of these diagnostics to hypersonic flows is a recent NATO review article that addresses absorption spectroscopy, planar laserinduced fluorescence (PLIF), molecular tagging velocimetry (MTV), focused laser differential interferometry (FLDI), light scattering (Rayleigh and Raman), coherent anti-Stokes Raman scattering (CARS), particle imaging velocimetry (PIV) and optical emission spectroscopy (OES) (Danehy et al. 2019). These advances have been enabled by advances in laser technology, including the invention of the tunable dye laser, advancements in high pulse energy solid-state laser technologies, efficient harmonic conversion, injection and narrow linewidth control, rapid multiple pulse capabilities, and femtosecond and picosecond laser technologies. These have been coupled with advances in digital camera and intensifier capabilities. Many of the earlier approaches are now being leveraged by the development of $\mathrm{MHz}$ rate pulse burst lasers, $\mathrm{kHz}$ rate femtosecond lasers and highspeed, intensified cameras that use rapid on-chip storage capabilities.

For this review, we will focus on methods that are enabled or enhanced by high repetition femtosecond lasers and by narrow linewidth $\mathrm{MHz}$ rate pulse burst lasers and produce time accurate measurements of local nonequilibrium and unsteady flow properties, where "time accurate" refers to sampling that is not time or multiple pulse averaged. These laser systems, together with the methods they are enabling and methods that are on the near horizon, have the potential to revolutionize our ability to acquire critical data for model development and code validation in high enthalpy hypersonic flows. Some also produce instantaneous images of flow properties that enhance intuitive understanding of complex interaction.

Commercially available femtosecond lasers typically utilize titanium sapphire as a gain medium since it has a very broad gain bandwidth, enabling the generation and amplification of sub-picosecond pulses. Typical pulse widths are 50 to 100 femtoseconds centered around $800 \mathrm{~nm}$ in the near infrared. Pulse outputs of the systems considered here are in the $7 \mathrm{~mJ}$ to $14 \mathrm{~mJ}$ range and pulse repetition rates are $1 \mathrm{kHz}$ to $10 \mathrm{kHz}$, with the pulse energy dropping at the higher repetition rates. Femtosecond ytterbium-based fiber lasers operating at $1030 \mathrm{~nm}$ are also available, with commercial amplified systems operating at pulse repetition rates from $100 \mathrm{kHz}$ to $1 \mathrm{MHz}$, but with lower pulse energies $(<1 \mathrm{~mJ})$ and longer pulse lengths ( $300 \mathrm{fsec})$. It may be useful to recall that for Gaussian "transform limited" pulses, the full width and half maximum uncertainty product of time and frequency is $\Delta t \cdot \Delta f=0.44$. Thus, 50 femtosecond pulses have a frequency width of $8.8 \times 10^{12}$ Hertz or, at $800 \mathrm{~nm}$, about $20 \mathrm{~nm}$. A $1 \mathrm{~mJ}, 50 \mathrm{fsec}$ pulse corresponds to $20 \mathrm{Giga}-$ Watts, so the femtosecond lasers are useful for nonlinear interactions due to their high peak power, but they are not useful for high-resolution spectroscopy due to the wide spectral bandwidth. The relationship between frequency width and pulse time indicates that if the frequency spectrum is constrained, for example, by a spectral filter, then the pulse length is increased. This provides flexibility in the pulse length and spectral performance of femtosecond lasers-their pulse lengths can be controlled by spectral filtering methods based either on dichroic spectral filters or diffraction gratings. Femtosecond lasers can be frequency up-converted with good efficiency due to the high intensity, and operation at second, third and fourth harmonics is easily achieved. They are also efficient at driving optical parametric oscillators and amplifiers, which enable broad tunability over the UV, visible and infrared. For hypersonics, femtosecond lasers have enabled femtosecond laser electronic excitation tagging (FLEET) (Michael et al. 2011; Miles et al.. 2018), femtosecond/picosecond hybrid coherent anti-Stokes Raman scattering (hybrid CARS) (Prince et al. 2006) and greatly enhanced two-photon absorption laser-induced fluorescence (TALIF) (Goehlich et al. 1998).

The other new laser capability is the burst mode or pulse burst laser (Wu et al. 2000; Wu 2000). This laser is capable of emitting high energy pulses at up to or even exceeding $1 \mathrm{MHz}$ rates, enabling rapid imaging of dynamic phenomena when paired with a high framing rate camera. Commercial pulse burst lasers are now available and their operational capabilities are being upgraded at a notable pace (Slipchenko et al. 2021). Typical individual pulses have a temporal width of $\sim 10 \mathrm{~ns}$ and recent progress has extended the burst duration to many milliseconds and pulse energies to hundreds of millijoules (Felver et al. 2020), enabling quasi-continuous imaging of hypersonic flows and combusting environments. Shortening the pulse to sub-picoseconds (Roy et al. 2014) extends the pulse burst laser to achieve more effective nonlinear optical diagnostics. The laser is based on Nd:YAG technology and operates at 1.064 microns, but it is efficiently frequency doubled to $532 \mathrm{~nm}$ and can be tripled to $355 \mathrm{~nm}$ or quadrupled to $266 \mathrm{~nm}$. It operates with a continuous wave source laser which is sliced and amplified through a series of amplifier stages. Since the source laser has a naturally very narrow linewidth, the output of the pulse burst laser has a single longitudinal mode and a very narrow linewidth. This differs significantly from standard Nd:YAG lasers that naturally operate with multiple longitudinal modes and must be injection locked to constrain operation to a single mode. The consequence is that the pulse burst laser is ideal for applications that require narrow linewidth such as filtered Rayleigh scattering (FRS) (Forkey et al. 1996) and holography. It can also serve as a pump for frequency tunable 
optical parametric oscillators (Jiang et al.. 2011), extending the burst capability to virtually any wavelength from ultraviolet to the far infrared. Recent work on operating with much shorter pulse lengths, on the order of 100 picoseconds, has enabled high repetition rate nonlinear processes including the development of picosecond laser electronic excitation tagging (PLEET). (Jiang et al. 2017a, b).

\section{Diagnostic approaches based on femtosecond lasers}

\subsection{Femtosecond laser electronic excitation tagging (FLEET)}

Femtosecond laser electronic excitation tagging (FLEET) takes advantage of the high intensity and short pulse length of the femtosecond laser to drive a high-order multi-photon interaction that leads to the dissociation of nitrogen in air, in pure nitrogen or in nitrogen containing gas mixtures. When the nitrogen subsequently recombines, the two nitrogen atoms initially form an excited electronic state of molecular nitrogen which fluoresces in the red to near infrared spectral region, corresponding to the "first positive" emission band of molecular nitrogen. Due to the time required for recombination and the rather long lifetime of the upper excited state, this fluorescence continues for many tens of microseconds. The region of excitation is well localized by the focused femtosecond laser, creating a well-defined line extending through the focal, or Rayleigh region of the laser. Depending on the focusing, the tagged line can be many centimeters long. That line then moves with the flow and subsequent imaging by a fast gated intensifier captures the line displacement as a function of the delay time between tagging and the intensifier "on" time. In this manner, the velocity of the flow can be precisely measured and any velocity fluctuations due to turbulence or shear become immediately evident due to the distortion of the line. The intensifier can be gated "on" multiple times to follow the same tagged line as it evolves with time, enabling imaging at $\mathrm{MHz}$ rates even with the tagging only occurring at the laser pulse repetition rate. Thus, "Lagrangian" measurements of flow properties are produced. In this manner, FLEET differs from vibrationally excited nitric oxide monitoring (VENOM) (Sanchez-Gonzalez et al. 2012), Raman excitation plus laser-induced fluorescence (RELIEF) (Noullez et al. 1997) and other approaches that require separate tagging and interrogation lasers.

Recent experiments performed at the AEDC Hypervelocity Wind Tunnel 9 have provided accurate measurements of flow velocity in freestream and boundary layer nitrogen flow at Mach 10, 14 and 18, at very low pressure (down to 0.35 Torr) and density $\left(3 \times 10^{-3} \mathrm{~kg} / \mathrm{m}^{3}\right.$ ) (Dogariu et al.. 2019a, 2021). These experiments have been enabled by the development of transportable instrumentation for FLEET velocimetry capable of withstanding the operating conditions of large-scale wind tunnel facilities.

Figure 1 shows FLEET measurements taken at Mach 18 in AEDC Tunnel 9. The composite image in Fig. 1a shows FLEET tagged lines captured at five successive time steps after a single-shot laser pulse tagging: 2, 7, 12, 17 and 22 microseconds. The thin tagged laser line is broadened by diffusion in the low pressure environment, but the center of the displaced line can be found to good accuracy by fitting the broadened profile with a Gaussian curve, as shown by the red dots in Fig. 1a. The repetition rate of the laser pulse tagging was $1 \mathrm{kHz}$, and measurements were taken over the $\sim 6 \mathrm{~s}$

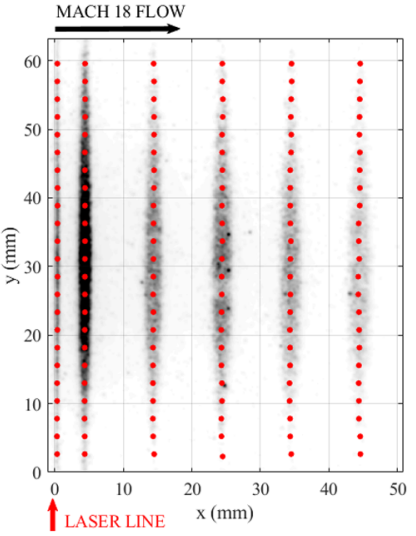

(a)

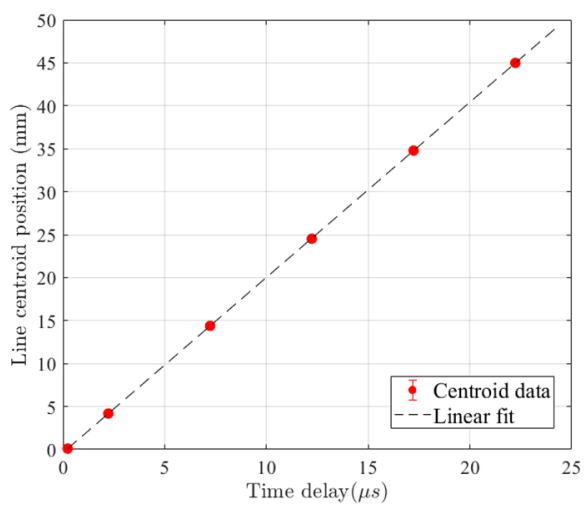

(b)

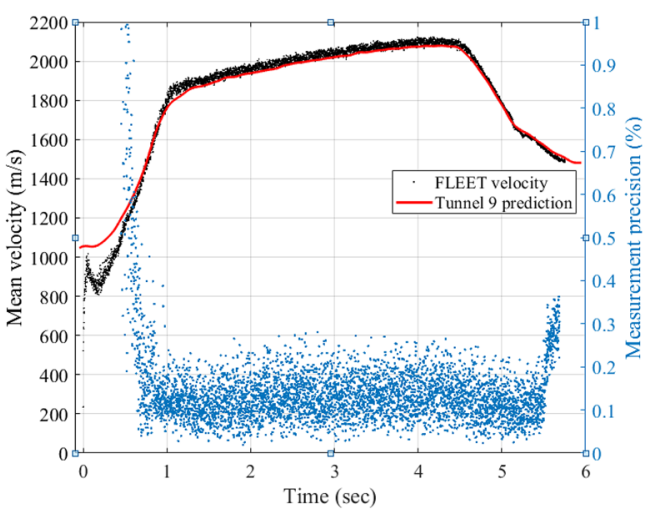

(c)
Fig. 1 a Single-shot composite raw FLEET image captured in Mach 18 freestream flow at AEDC Tunnel 9, b center line displacement versus time delay used for velocity estimation, $\mathbf{c}$ FLEET velocity and precision estimates throughout the Tunnel 9 operational envelope indicating a high dynamic range of measurements with greater than $0.2 \%$ precision in Mach 18 flow and good agreement with predictions based on plenum temperature data 
run time, which includes the $\sim 3.5 \mathrm{~s}$ fully developed Mach 18 flow and the transient flow. The almost perfect linear displacement with time fit shown in Fig. $1 b$ is a demonstration of a steady freestream flow velocity over the $22 \mu$ s observation interval. The time evolution of FLEET velocity measurements shown in Fig. 1c indicates that the velocity during the run changes due plenum gas heating. Figure $1 \mathrm{c}$ also shows that the precision of velocity measurements was better than $0.2 \%$ during the $\sim 3.5 \mathrm{~s}$ run, and the FLEET velocity estimates agreed very well with the predicted velocity from plenum temperature measurements.

An example of boundary layer velocity measurements using FLEET is shown in Fig. 2. Two sets of measurements (I) and (II) were taken during two different Mach 18 runs performed at the same operating parameters in order to capture the flow patterns inside (II) and outside (I) the boundary layer of the $1.5 \mathrm{~m}$ diameter test section of the tunnel. The two image sets (I) and (II) consist of 500 time-averaged single shots collected in fully developed Mach 18 nitrogen flow, and converted to velocity measurements. The velocity uncertainties in the outside region (I) are smaller than the data point in the figure. Uncertainties in the inner region (II) are indicative of the turbulent boundary layer. In Fig. 2, the mean streamwise velocity $(\bar{U})$ is normalized to the local edge velocity obtained from the first set of measurements (I). The measurement configuration has allowed recovering the boundary layer velocity profile as close as to $2 \mathrm{~cm}$ from the tunnel surface, and the measured boundary layer thickness of $~ 32 \mathrm{~cm}$ is in good agreement with the theoretical prediction (Dogariu et al. 2021).

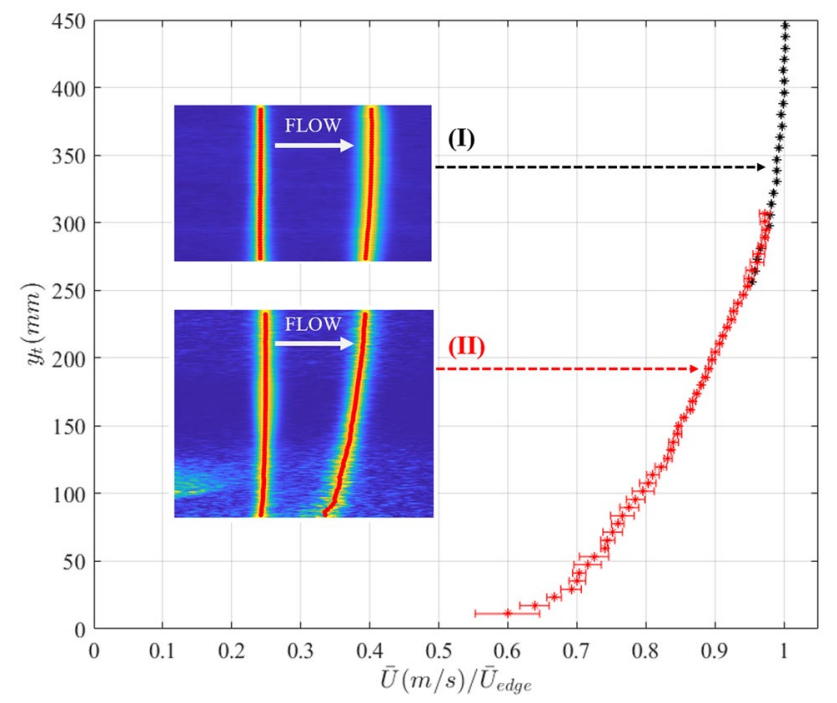

Fig. 2 FLEET velocity measurements in the wind tunnel sidewall boundary layer. Error bars represent one standard deviation of multiple velocity measurements taken within the Mach 18 tunnel operating envelope. The errors in the outer portion of the boundary layer (black diamonds) are smaller than the size of the diamonds
FLEET velocimetry has been also demonstrated in other complex flow environments, such as in combustion (DeLuca et al. 2017), in the NASA Langley high-pressure cryogenic tunnel (Burns et al. 2018a, 2018b), in bladeless wind turbines (Fisher et al. 2020a), in the Quiet Mach 6 tunnel at Purdue (Fisher et al. 2021), in a high-enthalpy arc-jet tunnel (UTA 2020) and in the wake of a cone at Mach 8 at Sandia (Zhang et al. 2019). Multiple line FLEET has been applied at Sandia (Zhang et al.. 2020) for measurements in an overexpanded unsteady supersonic jet.

Several refinements of this technique have addressed the concerns related to the energy deposition. Depending on the laser pulse energy, that heating associated with the FLEET tagging step can be on the order of $200 \mathrm{~K}$ within the tagged line (Edwards et al. 2015). This increase in temperature raises concern about possible perturbations to the flow measurement. Operating with the Ti:sapphire laser frequency doubled to $400 \mathrm{~nm}$ improves the tagging by reducing the pulse energy required and narrowing the width of the tagged line (Dogariu et al. 2019a). Selective two-photon absorptive resonance femtosecond laser electronic excitation tagging velocimetry (STARFLEET) (Jiang et al. 2016) has been developed to minimize the heating. STARFLEET moves the tagging wavelength into the ultraviolet at $202.25 \mathrm{~nm}$, a wavelength which overlaps a two-photon transition to a pre-dissociative state of molecular nitrogen. This resonant approach reduces the energy required for dissociation by a factor of 30 compared to FLEET at $800 \mathrm{~nm}$ and significantly reduces heating. Recent experiments at NASA have applied STARFLEET for measurements in cryogenic tunnels (Reese et al.. 2020).

\subsection{Hybrid femtosecond/picosecond coherent anti-stokes Raman scattering (Hybrid CARS)}

The four-wave mixing of coherent anti-Stokes Raman scattering (CARS) leaves molecules in their original states, so there is no intrinsic heating, as compared to FLEET, which relies on dissociation. Two pump beams that are separated in wavelength by the Raman shift coherently drive the selected molecules and a probe beam interacts with those molecules producing the CARS output beam. The CARS beam emerging from the sample region is highly directional due to phase matching, so background luminosity is easily rejected. Localization is achieved by crossing the pump beams in the sample region (Eckbreth 1996). Earlier work on CARS spectroscopy applications for temperature and species concentration used nanosecond laser systems in combustion environments (Eckbreth 1980) and more recently in high enthalpy arc facilities (Gülhan et al. 2018). For these applications, the operation has been at repetition rates of 10 to $30 \mathrm{~Hz}$ due to the pulse energies required to achieve intensities high enough to provide good signal strength. CARS suffers from 
a non-resonant background which involves a similar nonlinear interaction and thus satisfies the same phase matching and wavelength relationships. Due to coherent interference, this background is particularly troublesome for the measurement of other than major species and for the measurement of closely spaced spectral features. Suppression of this background in nanosecond applications is achieved by selective polarization, at the cost of reduced signal power.

The use of CARS for measurement of species mole fractions, temperature and nonequilibrium states has been revolutionized by the development of the hybrid femtosecond/ picosecond CARS approach (Prince et al. 2006; Pestov et al. 2007; Dogariu 2015; Miller et al.. 2010; Dedic et al. 2017; Dogariu and Pidwerbetsky 2012). The hybrid fs/ps approach can achieve CARS with a single femtosecond laser operating at kilohertz rates and suppresses the nonresonant background by temporal delay of the probe. The low energy $(\sim \mathrm{mJ})$ of the femtosecond pulse is offset by its short pulse length, leading to exceptionally high intensities and thus very efficient nonlinear pumping. By their nature, the femtosecond pulses are broadband, so they can be used to simultaneously drive many modes and even many molecular species into coherent oscillation. The proper Raman shifted wavelengths required for pumping are generated from the initial laser pulse by optical parametric mixing or by continuum light generation in hollow waveguides. Since the non-resonant interaction has essentially no lifetime and the pumped states all have coherent lifetimes much longer than femtoseconds, separating the timing of the pump and probe pulses eliminates the non-resonant background. Varying the time delay of the probe pulse allows sampling the coherent evolution of the excited states, and that temporal evolution can be converted into the Raman spectrum (Lucht et al. 2006). Alternatively, probing with a single narrow linewidth laser pulse and observing the spectrum with a spectrometer produces a single-shot measurement of a wide spectral region (Prince et al. 2006; Pestov et al. 2007). In this case, minimizing the time separation interval between the femtosecond pump beams and the probe beam is important to avoid coherent mode beating in the observed spectrum arising from the multiple time-evolving molecular states. Thus, the probe delay needs to be long enough to avoid nonresonant background and short enough to avoid significant mode beating. The delayed narrow linewidth probe pulse produces a broadband width, phase matched coherent output beam which is sent into a spectrometer and provides a spectral readout of the populations of all the coherently driven molecular states. The spectral resolution is limited by the linewidth of the probe pulse. In many cases, sufficient resolution can be achieved with a picosecond probe pulse that has a much narrower spectral width than the femtosecond pump pulses. The time delayed picosecond pulse can be created from a femtosecond pulse by spectral filtering (a narrower spectrum produces a longer pulse), so the same femtosecond laser can be used for all the required pulses (Dogariu 2015). Each laser pulse captures a broad spectrum, so the multiple molecular state populations associated with local species mole fractions as well as rotational and vibrational temperatures can be sampled at the laser pulse repetition rate.

Recent experiments at AEDC Tunnel 9 have applied this method to the measurement of the rotational and vibrational temperatures at a one $\mathrm{kHz}$ rate throughout the entire run times for multiple runs at Mach 10, 14 and 18, leading to the determination that the vibrational mode is far from equilibrium (Dogariu et al.. 2019b, 2021). The left panels of Fig. 3 show examples of the measured single-shot nitrogen rotational and vibrational spectra during a Mach 18 run. The vibrational spectrum shown in the top left panel of Fig. 3 contains rotational lines, so both the vibrational and the rotational temperatures can be extracted. The bottom left panel shows the pure rotational spectrum. A modified CARSFT (Palmer 1989) code was used to generate the best fits for the spectral shapes of the synthetic spectra and is shown in red in the left two panels of Fig. 3. Since the CARSFT routine does not account for the time delay between pump and probe, it cannot be used reliably to determine the temperature for hybrid fs/ps CARS, particularly for the rotational temperature due the fast decoherence of the rotational modes and strong dependence on the pulse delays. In order to use this code to obtain the temperatures for the experiments conducted at Tunnel 9, a relative calibration was performed by comparing the CARSFT simulations with spectra recorded in a heated oven with temperatures ranging from ambient to $1,400 \mathrm{~K}$. Best fits were achieved by adjusting the parameters included in the code, such as pulse bandwidths, saturation and pressure. The long coherence time of the vibrational modes led to good fits as shown in Fig. 3. At the very low rotational temperatures in the hypersonic flows in Tunnel 9, there are very few rotational modes active, so the decoherence is relatively slow. In this case, the calibration procedure used to determine the rotational temperature found that the CARSFT routine gives values within the experimental errors.

The time evolution of the vibrational temperature derived from the observed $\mathrm{N}_{2}$ Q-branch $(\Delta \mathrm{J}=0, \Delta= \pm 1)$ shown on the right side of Fig. 3 was obtained during a Mach 18 run. The combined experimental and fitting uncertainties are shown in the figure. The results found vibrational temperatures greater than $1100 \mathrm{~K}$ and rotational temperatures of $\sim 35 \mathrm{~K}$. The vibrational temperature increase seen during the three second run is due to an increase in plenum temperature, $\mathrm{T}_{0}$, shown by the red line. This lack of vibrational equilibration is a characteristic of rapid expansions that are typical of hypersonic ground test facilities and needs to be taken into account in the modeling for accurate agreement 

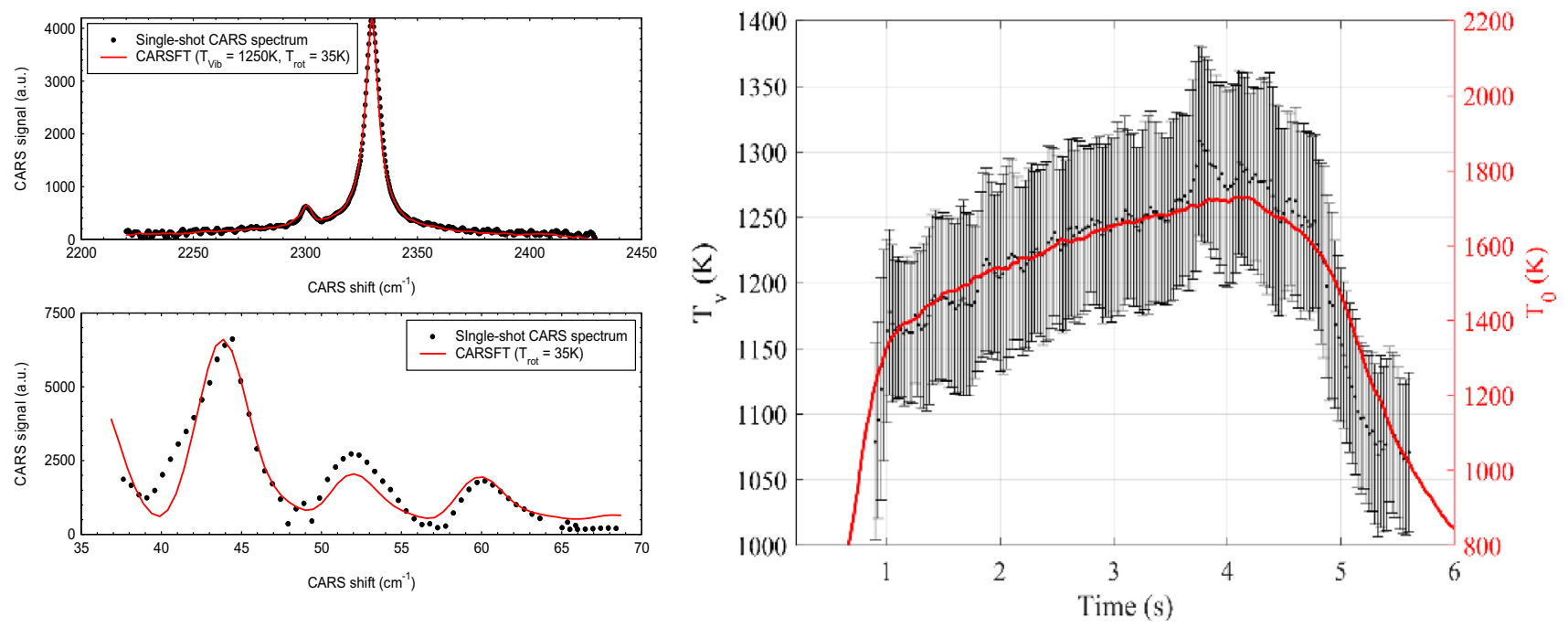

Fig. 3 Left: Vibrational (top) and rotational (bottom) hybrid fs/ps CARS spectra (black) and best fit nonequilibrium calibrated CARSFT numerical simulations (red) for Mach 18 flow in AEDC Tunnel 9. Right: Comparison of $1 \mathrm{kHz}$ rate hybrid fs/ps CARS vibrational tem-

(Reinert et al. 2020). Current work at Sandia is expanding the hybrid CARS to the measurement along a line, opening the door to spatially resolved measurements of rotational and vibrational temperatures as well as molecular species distributions (Chen et al.. 2020).

\subsection{Two-photon absorption laser-induced florescence (TALIF)}

Hybrid CARS and CARS in general rely on the presence of vibrational and rotation molecular states and are thus not applicable for measurements of atomic species; however, the presence of atomic species from dissociation is common in hypersonic flows and atomic species may be contaminants in high enthalpy ground test facilities. Standard laser-induced fluorescence of relevant atomic species is not possible in air since the wavelengths required are in the vacuum ultraviolet. This problem has been overcome by the combustion community through the development of two-photon absorption laser-induced fluorescence (Goehlich et al. 1998). In this case, the laser can operate at wavelengths that are not strongly absorbed by air, and through a two-photon interaction, can reach high lying atomic resonant states which subsequently fluoresce. Deriving quantitative values using this approach has been hampered by the nonlinear nature of the excitation, fluorescence quenching, ionization and stimulated emission, and, for gases with residual undissociated molecules, laser-induced dissociation. Moving to femtosecond pulse methods has alleviated the dissociation limitation by achieving the excitation of atomic species that are perature $\left(\mathrm{T}_{\mathrm{v}}\right)$ measurements (black) in the test section and plenum temperature $\left(\mathrm{T}_{0}\right)$ (red) versus time for a three second run plus turn on and turn off transients

present in the test gas faster than the dissociation can occur. Contrary to expectations, relatively efficient excitation of states with linewidths far narrower that the linewidth of the femtosecond pulse occurs due to the multi-photon process, where the red and blue skirts of the spectrum offset each other and add to the required narrow transition wavelength (Kulatilaka et al. 2012). An advantage of the femtosecond approach is that the commercial femtosecond lasers operate at higher repetition rates than nanosecond lasers and have excellent stability from one pulse to the next, so fluctuations associated with the multi-photon nature of the excitations are minimized. Quenching can be measured by capturing the fluorescence at two or more times after excitation (Settersten et al. 2006), allowing extrapolation to unquenched densities. Stimulated emission and ionization are residual problems that limit laser fluence. Stimulated emission can be monitored by measuring the backward emission along the excitation laser path (Agrup and Alden 1994), and ionization can be monitored by microwave scattering from free electrons (Dogariu and Miles 2011). Possible methods of calibration include seeding a small but well-known concentration of a noble gas such as xenon and simultaneously exciting those atoms, providing an ability to ratio the TALIF from the noble gas with that of the atomic species (Niemi et al. 2001). Both xenon and oxygen have two-photon resonances that are at almost the same wavelength (225.5 and $224.3 \mathrm{~nm}$ for xenon and $225.6 \mathrm{~nm}$ for oxygen). Krypton and atomic nitrogen and atomic hydrogen are close as well (204.2 for krypton, $206.6 \mathrm{~nm}$ for nitrogen and $205.1 \mathrm{~nm}$ for hydrogen). The full width at half maximum of a Gaussian $50 \mathrm{fsec}$ pulse 
at $266 \mathrm{~nm}$ is $\sim 2 \mathrm{~nm}$, so excitation of xenon and oxygen may be achieved simultaneously. Figure 4 shows the excitation and emission transitions for TALIF in atomic oxygen and nitrogen, together with the similar schemes for the atomic species used for TALIF calibration: $\mathrm{Xe}$ and $\mathrm{Kr}$, respectively. Recent measurements in the arc-jet facility at the University of Texas at Arlington have demonstrated the capability of fs-TALIF to measure atomic species in high enthalpy hypersonic flows (UTA press release 2020). Figure 5 shows TALIF fluorescence from naturally occurring xenon in air ( $~ 80$ parts per billion) following femtosecond two-photon

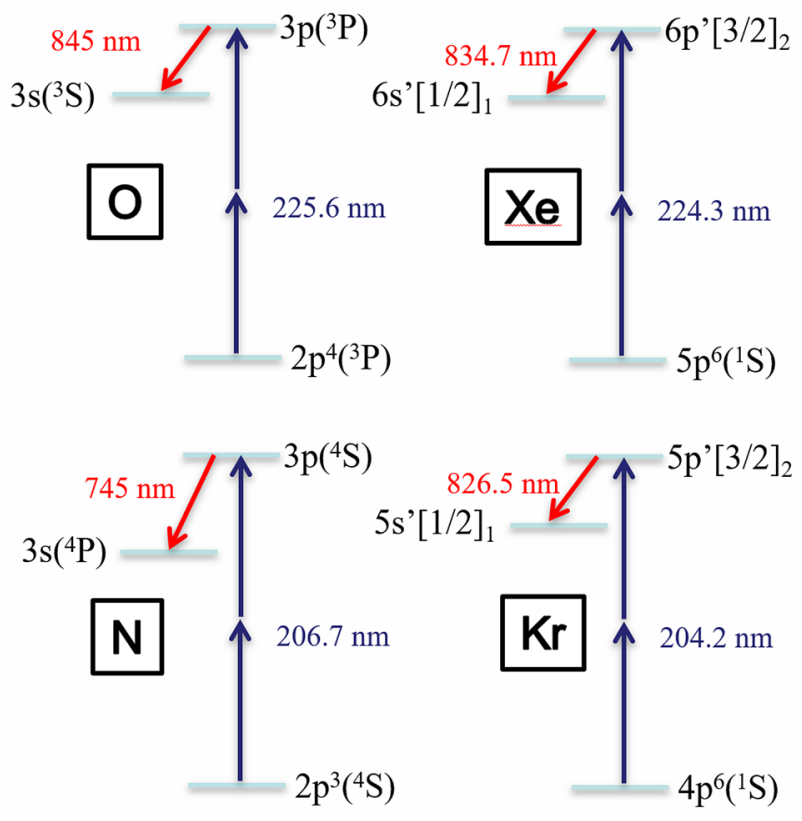

Fig. 4 TALIF schemes of atomic oxygen and nitrogen, together with xenon and krypton excitation at $252.48 \mathrm{~nm}$, demonstrating the high sensitivities that can be achieved.

\section{Pulse burst laser enabled methods}

The pulse burst laser enables numerous previously developed laser diagnostics to be implemented at high repetition rates and is particularly useful for acquiring data in short run time facilities and for the measurement of rapid interactions. A review of applications is provided by Thurow (2013) and more recently by Slipchenko et al. (2021)

\subsection{Filtered Rayleigh scattering}

The use of planar Rayleigh scattering for imaging supersonic and hypersonic flows provides instantaneous details of turbulent boundary layer structure, shock structure and shock wave/boundary layer interactions (Miles and Lempert 1990). Initial applications of $\mathrm{MHz}$ rate filtered Rayleigh scattering were for the imaging the temporal evolution of shock boundary layer interactions though Rayleigh scattering from carbon dioxide nano particles that formed in the core of low enthalpy supersonic and hypersonic flows. In this case, approximately $1 \%$ of $\mathrm{CO}_{2}$ was introduced into the air upstream of the plenum (Erbland et al. 1997; Wu 2000; Poggie et al. 2004) and as the flow expanded through the nozzle, the $\mathrm{CO}_{2}$ vapor condensed into a thin fog of $\mathrm{CO}_{2}$ nanoparticles in the core region, but not in the boundary layer due to the higher temperature in that region. Thus, the $\mathrm{CO}_{2}$ fog provided high contrast images showing the structure of the outer boundary layer and dynamic interactions with shocks and shock-induced separation. This approach takes advantage of the filtered Rayleigh capability by frequency doubling the pulse burst laser to $532 \mathrm{~nm}$ and placing an iodine vapor cell as a filter
Fig. 5 TALIF from natural xenon in room air at $\sim 80$ parts per billion. The line of excitation through the high intensity focal region of the $252.48 \mathrm{~nm}$ laser beam is seen in the "on resonance" image and not in the amplified "off resonance" image. Emission is at $823.5 \mathrm{~nm}$ in the IR. The weak peak in the off resonance image is due to non-resonant broadband emission from the air

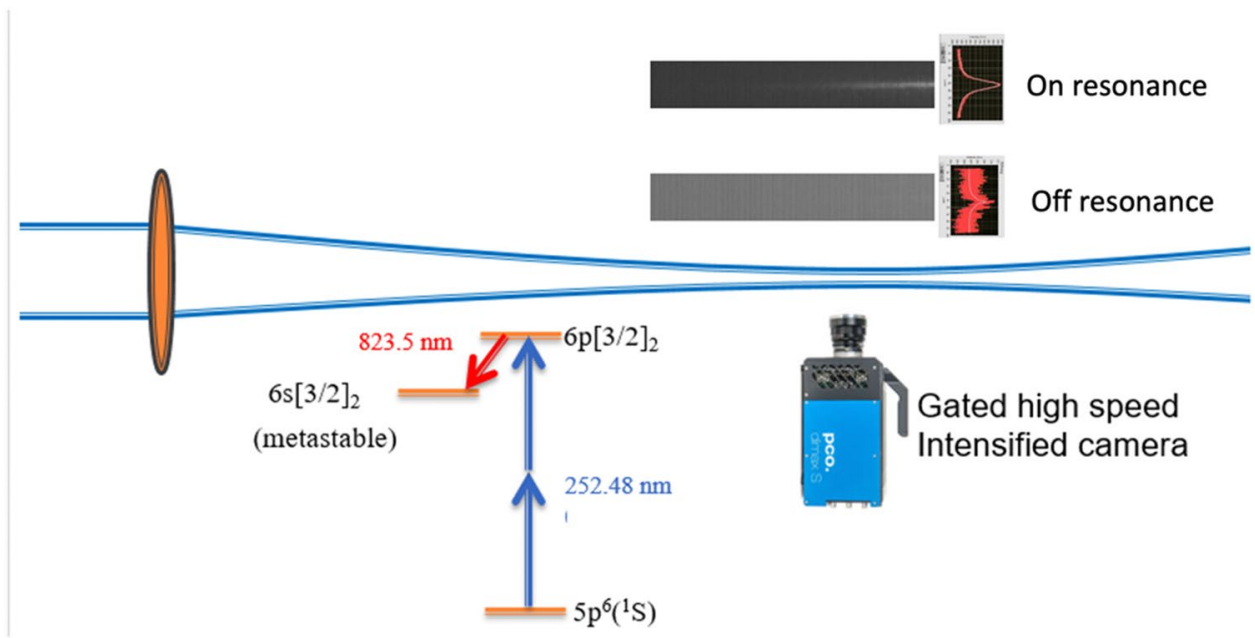


in front of the camera. (Huntley et al. 2000; Miles et al.. 2001) Iodine has numerous strong absorption lines that fall within the gain spectrum of the frequency doubled Nd:YAG laser. The narrow linewidth continuous wave seed laser is tuned so the second harmonic wavelength overlaps one of these strong iodine absorption lines. By illuminating the flow at an angle such that the light from the moving $\mathrm{CO}_{2}$ particles is Doppler shifted out of the iodine absorption, the background from the stationary windows and walls that otherwise obscures the flow images is eliminated while the Doppler shifted light scattered from the flow passes through the filter and is imaged by the camera. Figure 6 shows sequential images taken with this approach.

The challenge for the Rayleigh measurement of density and other parameters in unseeded hypersonic flows is to achieve sufficient pulse energies to provide good signal to noise from the low densities associated with hypersonic ground test facilities. The Rayleigh scattering cross section is greatly enhanced in the ultraviolet, so increases in pulse energy, extension to the ultraviolet and implementation of other filters to block out background scattering including interferometric filters (Cutler et al.. 2020) and atomic vapors such as mercury at $254 \mathrm{~nm}$ and optically pumped barium at $355 \mathrm{~nm}$ (Hetlage and Limbach 2021) may enable this capability.

By tuning the laser across the absorption band of the vapor filter, filtered Rayleigh scattering can be used for imaging velocity, temperature and density by deconvolving the measured profile to give the lineshape and frequency shift of the Rayleigh scattering, (Forkey et al. 1998) This approach requires many image frames to be taken and pixel resolved deconvolution, so it is not useful for rapidly varying flows or short run time facilities. Single-shot imaging of specific flow properties can be achieved by tuning the laser relative to the vapor filter absorption spectrum, changing the collection angle or changing the vapor pressure (Miles et al.. 2001). For example, when the Rayleigh scattering linewidth matches the filter linewidth, the scattering is independent of temperature (Feng et al. 2020) leading to a measurement of pressure, assuming an ideal gas. By tuning the laser to the edge of the vapor absorption profile, the scattering is sensitive to velocity (Thurow et al. 2005). That velocity sensitivity can be enhanced by changing the vapor absorption line profile with the addition of an inert gas that collision broadens the line (Elliott and Beutner 1999).

\subsection{MHz rate laser-induced fluorescence (LIF)}

The extension of the pulse burst laser to variable wavelength operation opens the door to its use for laser-induced fluorescence applications. The only candidate for LIF in pure air is oxygen, which for atmospheric temperatures requires a laser operating in the ultraviolet overlapping the Schumann Runge bands at wavelengths below $200 \mathrm{~nm}$. This has been achieved using ArF lasers operating at $193 \mathrm{~nm}$ (Laufer et al. 1990), but so far that wavelength region has not been reached with the pulse burst laser. If nitric oxide is naturally present from the high temperature in the plenum or from post-shock heating or it is seeded into the flow, it can be used for LIF with the frequency tunable MHz laser operating at $226 \mathrm{~nm}$ (Jiang et al.. 2011). An example is the stereo imaging achieved in the 31-Inch Mach 10 Air Tunnel at NASA Langley (Medford et al.. 2011). In this case, the air was seeded with nitric oxide from a spanwise slot on a wedge model and the evolution of the seeded air was followed after passing over a small cylindrical protuberance with stereo imaging at up to $500 \mathrm{kHz}$. The high repetition rate may also enable the vibrationally excited nitric oxide monitoring (VENOM) technique (Sanchez-Gonzalez et al. 2012).

\subsection{Picosecond laser electronic excitation tagging (PLEET)}

Significantly shortening the pulse to sub-nanoseconds while maintaining the pulse energy enables the $\mathrm{MHz}$ pulse burst system to achieve flow tagging through PLEET (Jiang et al. 2017a, b). This approach is similar to FLEET and was developed to overcome the lack of very high rep rate, high pulse energy $(\sim 1 \mathrm{~mJ}$ per pulse which is what is required for FLEET) femtosecond lasers. PLEET requires about 30 times more energy per pulse than FLEET, since the pulses are approximately 1000 times longer, and thus have much lower
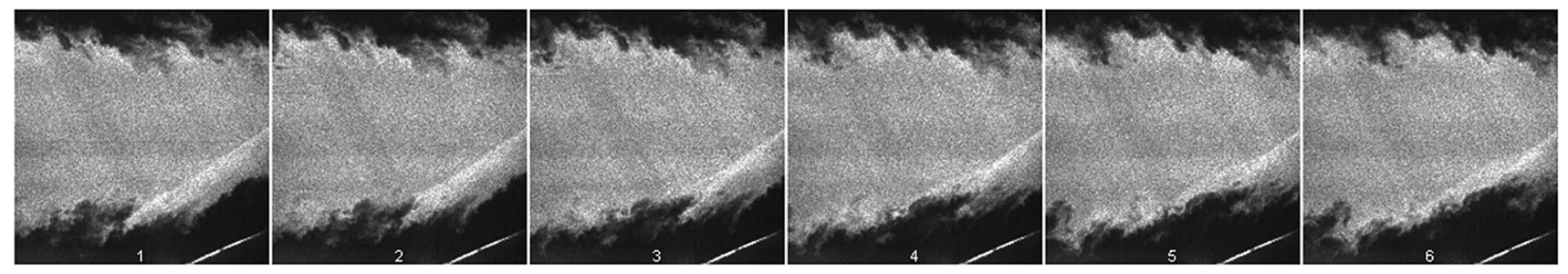

Fig. 6 Mach 2.5 air flow over a 24॰ wedge taken at 500000 frames per second with the pulse burst laser and an iodine "notch" filter. Images are sequenced in $2 \mu$ s time steps from left to right. Flow is from left to right, and light is scattered from $\mathrm{CO}_{2}$ particles 
peak intensity. Significantly more heating of the tagged region occurs, and strong short-lived emission in the ultraviolet corresponding to the nitrogen "second positive" bands occurs over the first $500 \mathrm{nsec}$ or so, followed by long lifetime "first positive" red and infrared emission characteristic of FLEET that is used for the displacement imaging. The tagged regions continue to fluoresce for longer times than FLEET, which may be associated with the higher temperature and higher energy deposition. Applications at $100 \mathrm{kHz}$ in a Mach 6 Ludwieg tube at the Air Force Research Lab (Hsu et al.. 2020) and $25 \mathrm{kHz}$ measurements in transonic facilities at NASA have been conducted (Burns et al. 2018a, 2018b).

\section{Future capabilities}

The continued development of laser technologies will significantly impact hypersonic diagnostics and potentially bring some of these methods to flight data acquisition systems. These advances will further the goal of capturing true timefrozen data at rates high enough to follow dynamic processes at locations that are relevant to vehicle design and to the determination of critical physical processes. For example, the development of $100 \mathrm{kHz}$ to $\mathrm{MHz}$ rate, high pulse energy femtosecond laser systems will enable the application of FLEET, fs/ps CARS and TALIF at those repetition rates (Smyser et al. 2019). The extension of MHz rate lasers to this femtosecond regime unites both the femtosecond diagnostics with the $\mathrm{MHz}$ sampling capability, enabling rapid measurements in dynamic environments including both external hypersonic flows and internal flows for air breathing, SCRAM jet powered platforms. These approaches involve combinations of femtosecond seeding, amplification with broadband Nd:glass, and pulse shape control and represent yet another step in the laser technology that promises to significantly advance diagnostic capabilities. Preliminary demonstrations of this capability have been achieved both for FLEET (Fisher et al. 2020b) and hybrid CARS (Smyser et al. 2020).

An increase in the pulse energy of the pulse burst lasers will enable Rayleigh measurements of low density gases. Higher repetition rates may enable microsecond time-frozen volume imaging with spatially swept beams. Rapidly frequency swept pulsed lasers will enable other nonlinear process such as frequency chirped coherent Rayleigh/Brillouin scattering to be implement for the measurement of velocity and temperature. (Gerakis et al.. 2021). Multi-wavelength pulsed lasers may enable simultaneous calibration and single-pulse imaging of velocity fields and thermodynamic properties. Higher spectral purity lasers will further atomic and molecular filter capabilities.
The integration of atomic filters into the detection systems together with the development of higher pulse energy, narrow linewidth tunable pulse burst lasers provide new opportunities for hypersonic diagnostics. Two such approaches are currently being explored - slow light imaging spectroscopy (SLIS) (Miles and Dogariu 2020; Dogariu and Miles 2018) for imaging rotational Raman features and filtered low-angle Thomson scattering (FLATS) (Miles et al. 2020) for detecting low density ionization near leading edges and in boundary layers of hypersonic test articles. SLIS takes advantage of the delayed propagation of light through atomic vapor cells when the light wavelength falls close to an atomic resonance (Hau et al. 1999; Wang et al. 2000). The wavelengths of Rayleigh, Raman and Thomson scattering all follow the wavelength of the excitation laser source. Therefore, if the laser source wavelength is changed, their wavelengths are changed, so with a tunable laser, the Rayleigh, Raman or Thomson wavelengths can be tuned to fall close to an atomic vapor resonance. Other features of Rayleigh, Raman and Thomson scattering are that the scattering only occurs during illumination and that it does not suffer from quenching. In this way, Rayleigh, Raman and Thomson scattering differ substantially from laser-induced fluorescence which has an excited state lifetime and has its fluorescence significantly affected by quenching. The primary difficulty with Rayleigh, Raman and Thomson scattering is the very low scattering cross sections, so methods that remove background interference and enhance light collection may have significant impact on data acquisition capabilities for hypersonics. This is especially important for data acquisition in short run time shock and expansion tubes where long time averaging is not possible and for capturing dynamic phenomena and turbulent flow properties.

\subsection{Slow light imaging spectroscopy (SLIS)}

The two features of Rayleigh, Raman and Thomson scattering, frequency tunability and time synchronization, enable slow light imaging. Typical delays that can be achieved through atomic vapor cells without significant loss can be more than $20 \mathrm{~ns}$, so if the illumination time is shorter than that, then the scattering light pulse delay will be greater than the pulse time and the delayed light can be easily distinguished by an intensifier time gated camera. The atomic vapor cell does not affect the propagation vectors of the delayed light, so images are preserved. This opportunity is particularly useful for rotational Raman imaging, since rotational Raman is significantly stronger than vibrational Raman, and Raman scattering is capable of providing measurements of temperature, species concentrations and nonequilibrium. The low scattering cross section, the proximity of strong Rayleigh and background laser scattering, the interleaved rotational spectral lines from other constituents 
and the need for a spectrometer have previously limited the ability to image these spectral features. The acceptance bandwidth of the atomic filter is a few $\mathrm{GHz}$, so assuming the laser is injection locked (narrow band), individual rotational Raman lines can be imaged. The product of collection solid angle and collection aperture (etendue) can be orders of magnitude greater than spectrometers. Candidate elements for atomic filters are cesium, potassium, rubidium, sodium and mercury. There is a fundamental trade-off between transmission and delay: longer delays result in lower transmission. The Raman scattering is greatly enhanced in the ultraviolet, so operation using atomic resonances in the ultraviolet is preferred, suggesting mercury at $245 \mathrm{~nm}$ or UV transitions in alkalis. Figure 7 shows the predicted delay for a $5 \mathrm{~ns}$ long transform limited pulse tuned to a transmission window between isotopic lines of mercury and passing through a 2 " long atomic mercury vapor filter with the side arm temperature held at $100 \mathrm{C}$. A delay of up to $50 \mathrm{nsec}$ is predicted with $60 \%$ transmission (Rad et al. 2020). Better performance may be achievable with isotopically purified mercury.

\subsection{Filtered low-angle Thomson scattering (FLATS)}

Filtered low-angle Thomson scattering provides a method to suppress background interference and extend the sensitivity range of Thomson scattering. It takes advantage of the "coherent" nature of Rayleigh/Brillouin and Thomson scattering compared to the "incoherent" nature of Raman scattering. This difference arises due to the fact that Raman scattering leads to a change in the population of molecular energy states, and thus, there is no coherence associated with the scattering process. Raman scattered photons have random phase and the frequency shift from the illumination laser is set by the energy levels involved. Rayleigh/ Brillouin and Thomson scattering do not lead to a change in energy states, so the scattered photons retain the phase of the incident photons. Frequency shifts are associated with the observed net phase modulation from the laser source to the detector and arise from the motion of the scattering entities (atom, molecule, ion or electron) leading to broadening. In the forward direction, the observed net phase modulations of all scattered photons are the same and no broadening occurs from the thermal motion. When collected at 90 degrees to the illumination direction, the conventional collection configuration, the electron Thomson scattering linewidth extends beyond the rotational Raman shifts due to the very low mass and, consequently, the high thermal velocity of the electrons (Fig. 8). Measurement of electron density and temperature requires extracting the magnitude and lineshape of the electron Thomson scattering. Typical electron densities surrounding vehicles flying at Mach 13 are below $10^{13} / \mathrm{cc}$ (Surzhikov 2018), making the measurement extremely difficult due to the overlapping Raman lines from the much higher densities of neutral molecular species.

The narrowing of the scattering in the forward direction enables the selective narrowing of the Thomson scattering relative to the Raman lines and thus the separation of the

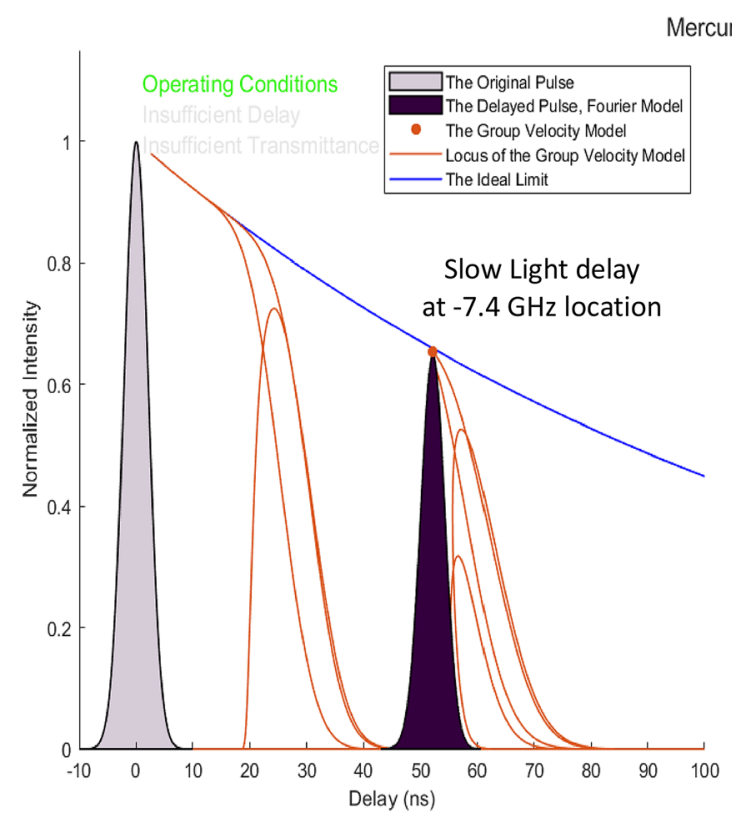

Fig. 7 Predicted delay for a 5 nsec pulse passing through a 2" long mercury vapor cell. The left panel shows the variation in delay as the laser is tuned across the mercury vapor absorption features and

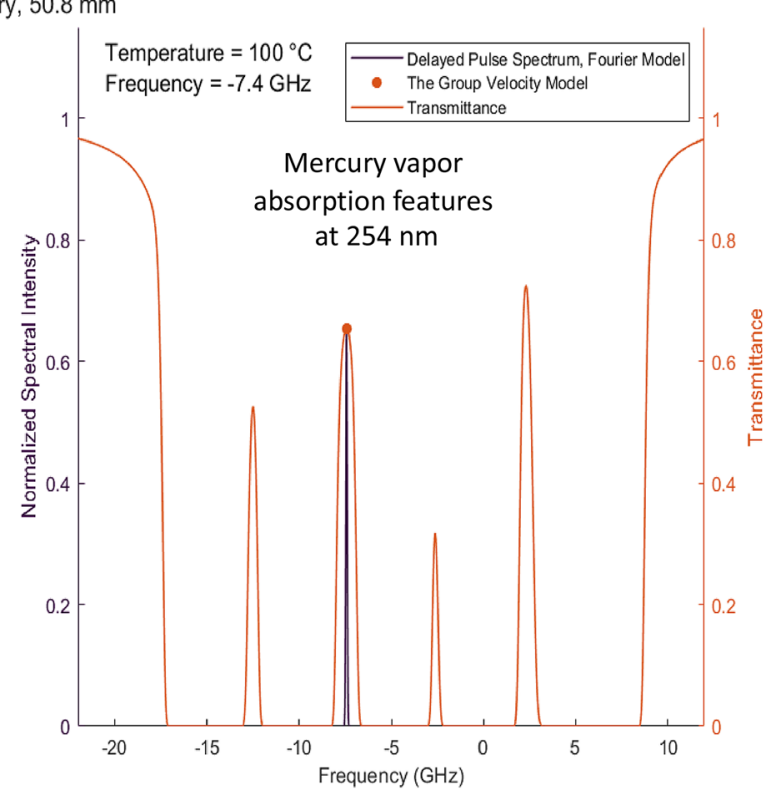

the black highlight shows the delay when the laser frequency falls between isotopic absorption lines as shown by the black line on the right hand panel 
Fig. 8 Spectral features of Rayleigh/Brillouin, Raman, Thomson and Mie scattering with conventional 90 degree scattering detection. Forward scattering narrows the Thomson and Rayleigh/Brillouin lines while leaving the Raman spectrum unchanged

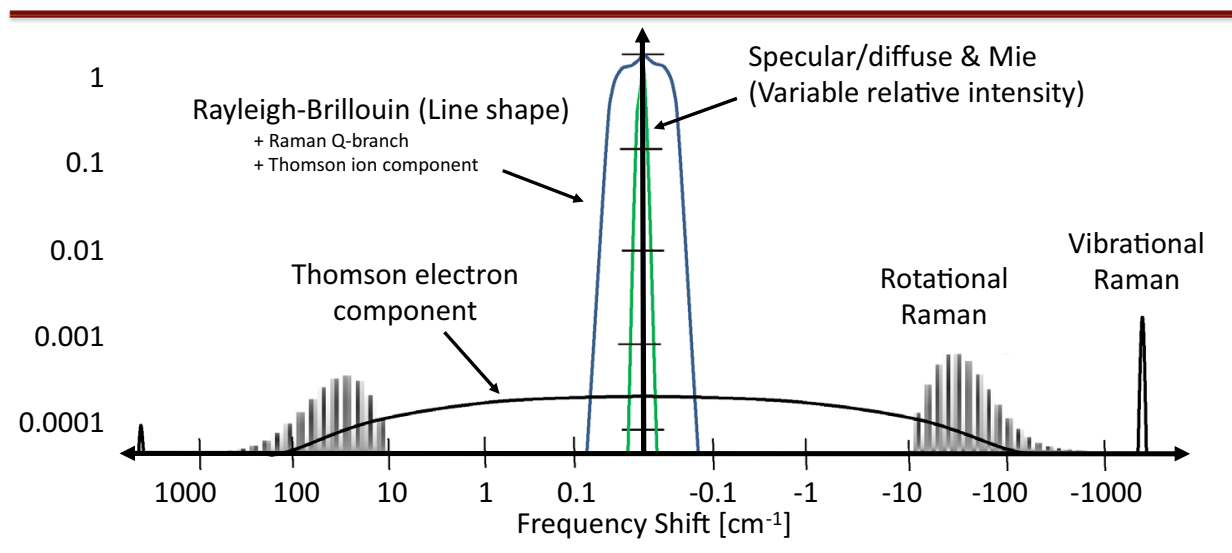

Thomson from the Raman. The Rayleigh/Brillouin scattering and ion Thomson scattering are also narrowed by the same process, so inserting an atomic filter and tuning the illumination laser to the atomic filter absorption feature eliminates the Rayleigh/Brillouin and ion Thomson scattering and passes the Raman and electron Thomson scattering (Zaidi et al. 2002; Lee and Lempert 2002). The spectrum can then be analyzed by passing that light into a spectrometer. Since the Thomson scattering is well separated from the Raman, overlap is avoided and the Thomson scattering spectrum and amplitude can be directly recorded. Background plasma luminosity is broadband, and narrowing of the Thomson scattering improves the rejection of that broadband light by spectral filtering. The spectrometer can be eliminated by configuring the atomic cell as a prism to disperse the Thomson scattering and enable measurement of the Thomson scattering spectrum (Finkelstein et al. 1998). With the laser tuned to the atomic line center, the background laser scattering, Mie scattering, Rayleigh and ion Thomson scattering are blocked and only the electron Thomson and Raman scattering pass. The dispersion is strongest near the atomic resonance, so the electron Thomson scattering is strongly dispersed, whereas the Raman scattering only weakly dispersed. Figure 9 shows the configuration for the capture of forward scattering and the subsequent spectral filtering using an atomic vapor prism.

In order to enhance this effect, a multi-prism cell can be employed. The Thomson scattering cross section is $\sigma=7.94 \times 10^{-26} \mathrm{~cm}^{2} / \mathrm{sr}$, independent of wavelength, so operation in the near infrared is preferred in order to minimize the Rayleigh and Raman/Brillouin background. Candidate atomic vapors are rubidium at $780.0 \mathrm{~nm}$ and $794.7 \mathrm{~nm}$, potassium at $766.5 \mathrm{~nm}$ and $769.9 \mathrm{~nm}$, and cesium at $852.1 \mathrm{~nm}$ and $894.3 \mathrm{~nm}$.

\section{Summary}

New approaches are revolutionizing the diagnostic capabilities for hypersonic ground test facilities, driven primarily by the availability of high-performance femtosecond and pulse burst laser capabilities. Molecular tagging has enabled the tracking of flow for measurements of velocity and acceleration as well as for the capture of turbulent structure and boundary layer profiles. Single-shot broad spectral
Fig. 9 Configuration for FLATS removal of Raman background and measurement of electron density and temperature with at atomic vapor prism

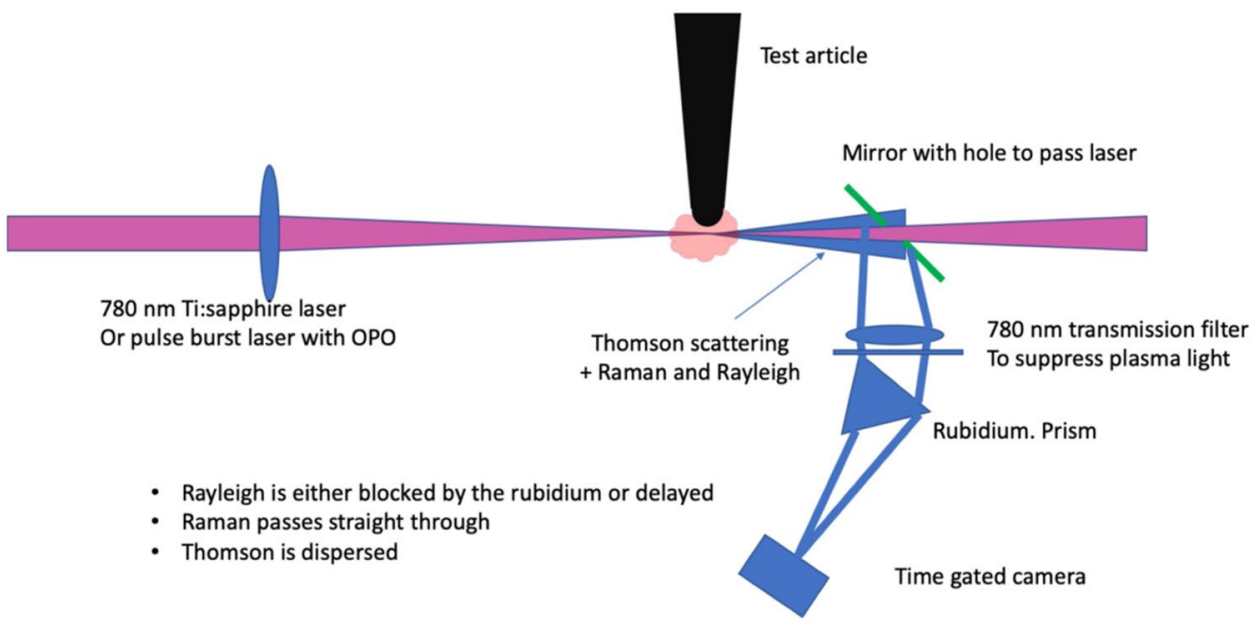


width femtosecond/picosecond CARS is providing real-time measurements of species mole fractions as well as localized rotational and vibrational temperatures. Atomic species are being tracked by TALIF, which is particularly useful at the low static pressures encountered in hypersonic facilities, since quenching rates are minimized. Seeding the flow with a small concentration of a noble gas provides a method for calibration. The extension of the pulse burst laser to frequency tunable narrow linewidth operation through the implementation of seeded optical parametric oscillators has opened the door to rapid LIF and potentially to the use of atomic filters for measurements of Raman and Thomson scattering at $\mathrm{MHz}$ rates. Extending the tunability range farther into the ultraviolet may enable rapid imaging of temperature and oxygen density through Schumann Runge LIF methods. Achieving picosecond and femtosecond pulses at $\mathrm{MHz}$ rates opens up the option for high rate nonlinear optical diagnostics, including flow molecular flow tagging. The continued development of laser capabilities along with detection methods shows great promise for more near-term advances based on rotational Raman and Thomson scattering.

Acknowledgements The authors thank multiple funding sources for support of their work on the development of diagnostics for hypersonics over the past many years including AFOSR, ONR, ARO and NASA and through SBIR programs with MetroLaser, Inc., and Spectral Energies, Inc. Current research on SLIS and FLATS is being supported by AFOSR under Dr. Sarah Popkin and by ONR under Dr. Eric Marineau. Work on the application of FLEET and hybrid fs/ps CARS in Tunnel 9 was supported by an SBIR contract from AEDC through Plasma TEC. Inc. together with Speckodyne, Corp.

Open Access This article is licensed under a Creative Commons Attribution 4.0 International License, which permits use, sharing, adaptation, distribution and reproduction in any medium or format, as long as you give appropriate credit to the original author(s) and the source, provide a link to the Creative Commons licence, and indicate if changes were made. The images or other third party material in this article are included in the article's Creative Commons licence, unless indicated otherwise in a credit line to the material. If material is not included in the article's Creative Commons licence and your intended use is not permitted by statutory regulation or exceeds the permitted use, you will need to obtain permission directly from the copyright holder. To view a copy of this licence, visit http://creativecommons.org/licenses/by/4.0/.

\section{References}

Agrup S, Alden M (1994) Two-photon laser-induced fluorescence and stimulated emission measurements from oxygen atoms in a hydrogen/oxygen flame with picosecond resolution. Opt Commun 113:315-323. https://doi.org/10.1016/0030-4018(94)90618-1

Alexandros G, Junhwi B, Robert R, Mikhail SN (2021) Demonstration of single shot laser velocimetry with coherent Rayleigh-Brillouin scattering 19-21 January 2021. AIAA Scitech 2021 Forum https:// doi.org/10.2514/6.2021-0224

Andreas E, Jiajian Z, Xuesong Li, Johannes K (2017) Advanced laserbased techniques for gas-phase diagnostics in combustion and aerospace engineering. Appl Spectrosc 3:341-366. https://doi. org/10.1177/0003702817690161

Arthur D, Dogariu Laura E, Smith Michael S, John L, Miles Richard B (2019) Single shot temperature measurements using coherent anti-stokes Raman scattering in Mach 14 flow at the hypervelocity AEDC tunnel 9. AIAA Pap No. https://doi.org/10.2514/6. 2019-1089

Arthur D (2015) Detection systems and methods using coherent antistokes Raman spectroscopy. US. patent \#9,163,988, Issued October 20, 2015.

Burns RA, Peters CJ, Danehy PM (2018a) Unseeded velocimetry in nitrogen for high-pressure, cryogenic wind tunnels, part I: femtosecond-laser tagging. Meas Sci Technol 29(11):115302

Burns RA, Danehy PM, Jiang NB, Slipchenko MN, Felver J, Roy $\mathrm{S}$ (2018b) Unseeded velocimetry in nitrogen for high-pressure cryogenic wind tunnels: part II Picosecond-laser tagging. Meas Sci Technol 29(11):115203. https://doi.org/10.1088/1361-6501/ aade 15

Chen TY, Goldberg BM, Patterson BD, Kolemen E, Ju Y, Kliewer CJ (2020) 1-D imaging of rotation-vibration non-equilibrium from pure rotational ultrafast coherent anti-Stokes Raman scattering. Optics Lett 45(15):4252-4255

Cutler Andrew D, Keith R, Sukesh R et al (2020) 100-kHz Interferometric Rayleigh Scattering for multi-parameter flow measurements. Opt Express 28(3):3025-3040. https://doi.org/10.1364/ OE.380934

Danehy PM, Weisberger J, Johansen C, Reese D, Fahringer T, Parziale NJ, Dedic C, Estevadeoral J, and Cruden BA, (2019) Nonintrusive measurement techniques for flow characterization of hypersonic wind tunnels. NATO S and T Report, STO-ENAVT-325. https://doi.org/10.14339/STO-EN-AVT-325-04-PDF

De Cesare M, Savino L, Ceglia G, Alfano D, Di Carolo F, French AD, Rapagnani D, Gravina S, Cipulloa A, Del Vecchio A, Di Leva A, D'Onofriob A, Galietti U, Gialanella L, Terrasi F (2020) Applied radiation physics techniques for diagnostic evaluation of the plasma wind and thermal protection system critical parameters in aerospace re-entry. Prog Aerosp Sci. https://doi.org/10.1016/j.paerosci.2019.06.001

Dedic CE, Meyer TR, Michael JB (2017) Single-shot ultrafast coherent anti-Stokes Raman scattering of vibrational/rotational nonequilibrium. Optica 4(5):563-570. https://doi.org/10.1364/ OPTICA.4.000563

DeLuca NJ, Miles RB, Jiang N, Kulatilaka WD, Patnaik AK, Gord JR (2017) FLEET velocimetry for combustion and flow diagnostics. Appl Opt 56:8632-8638

Dogariu A, Miles RB (2011) Detecting localized trace species using Radar REMPI. Appl Opt 50(4):A68-A71. https://doi.org/10. 1364/AO.50.000A68

Dogariu A, Pidwerbetsky A (2012) Coherent anti-stokes Raman spectroscopy for detecting explosives in realtime. Proc SPIE. https://doi.org/10.1117/12.919568

Dogariu A, Dogariu L, Smith M, MacManamen B, Lafferty J, Miles R (2021) Velocity and temperature measurements in Mach 18 nitrogen flow at tunnel 9. AIAA Pap. https://doi.org/10.2514/6. 2021-0020

Dogariu A, Miles R. B (2018) Slow light imaging spectroscopy with a passive atomic filter. Conference on lasers and electro-optics, OSA technical digest (online) (Optical Society of America, 2018, paper STu4P.1

Dogariu Laura E, Arthur D, Miles Richard B, Smith Mike S, Marineau Eric C (2019) Femtosecond laser electronic excitation tagging velocimetry in a large-scale hypersonic facility. AIAA J 57(11):4725-4737. https://doi.org/10.2514/1.J057759

Eckbreth AC (1980) CARS Thermometry in Practical Combustors. Combust Flame 39(2):133-147. https://doi.org/10.1016/00102180(80)90013-9 
Eckbreth A (1996) Laser diagnostics for combustion temperature and species in unsteady combustion. CRC Press, Dordrecht

Edwards MR, Dogariu A, Miles RB (2015) Simultaneous temperature and velocity measurements in air with femtosecond laser tagging. AIAA J 53(8):2280-2288. https://doi.org/10.2514/1. J053685

Elliott GS, Beutner TJ (1999) Molecular filter based planar Doppler velocimetry. Prog Aerosp Sci 35(8):799-845. https://doi.org/10. 1016/S0376-0421(99)00008-1

Erbland PJ, Baumgartner ML, Yalin AP, Etz MR, Muzas B, Lempert WR, Smits A, Miles RB (1997) Development of planar diagnostics for imaging Mach 8 flowfields using carbon dioxide and sodium seeding. AIAA Pap No. https://doi.org/10.2514/6. 1997-154

Felver J, Slipchenko MN, Braun EL, Meyer TR, Roy S (2020) Highenergy laser pulses for extended duration megahertz-rate flow diagnostics. Opt Lett 45(16):4583-4586. https://doi.org/10.1364/ OL.400831

Feng D, Goldberg B, Shneider MN, Miles RB (2020) Optimization of Filtered Rayleigh Scattering for the Measurement of Pressure and Temperature. Combust Sci Technol. https://doi.org/10.1080/ 00102202.2020 .1822345

Finkelstein ND, Yalin AP, Lempert WR, Miles RB (1998) Dispersion Filter for Spectral and Spatial Resolution of Pure Rotational Raman Scattering. Opt Lett 23(20):1615-1617. https://doi.org/ 10.1364/OL.23.001615

Fisher JM, James B, Meyer Terrence R et al (2020a) Application of femtosecond laser electronic excitation tagging (FLEET) velocimetry in a bladeless turbine. Meas Sci and Technol 31(6):064005

Fisher JM, Smyser ME, Slipchenko MN, Roy S, Meyer TR (2020b) Burst-mode femtosecond laser electronic excitation tagging for kHz-MHz seedless velocimetry. Opt Lett 45(2):335-338. https:// doi.org/10.1364/OL.380109

Fisher JM, Chynoweth BC, Smyser ME, Webb AM, Slipchenko MN, Jewell JS, Meyer TR, Beresh SJ (2021) Femtosecond laser electronic excitation tagging velocimetry in a Mach six quiet tunnel. AIAA J 59(2):768-772

Forkey JN, Finkelstein ND, Lempert WR, Miles RB (1996) Demonstration and characterization of filtered Rayleigh scattering for planar velocity measurements. AIAA J 34(3):442-448. https://doi.org/ 10.2514/3.13087

Forkey JN, Finkelstein ND, Lempert WR, Miles R (1998) Accuracy limits for planar measurements of flow field velocity, temperature and pressure using filtered Rayleigh scattering. Exp Fluids 24(2):151-162. https://doi.org/10.1007/s003480050162

Goehlich A, Kawetzki T, Dobele HF (1998) On absolute calibration with xenon of laser diagnostic methods based on two-photon absorption. J Chem Phys 108(22):9362-9370. https://doi.org/10. 1063/1.476388

Gülhan A, Esser B, Koch U, Fischer M, Magens E, Hannemann V (2018) Characterization of high-enthalpy-flow environment for ablation material tests using advanced diagnostics. AIAA J. https://doi.org/10.2514/1.J056312

Hanson Ronald K, Spearrin Mitchell R, Goldenstein Christopher S (2016) Springer spectroscopy and optical diagnostics for gases. Springer international publishing, Cham

Hau LV, Harris SE, Dutton Z, Behroozi CH (1999) Light speed reduction to 17 metres per second in an Ultracold atomic gas. Nature 397:594-598. https://doi.org/10.1038/17561

Hetlage Madison E, Limbach Christopher (2021) Combined Rayleigh and Raman Airborne temperature lidar at $355 \mathrm{~nm}$ with a non-maxwellian barium vapor filter. AIAA Scitech 2021 Forum. https:// doi.org/10.2514/6.2021-1377

Hsu Paul S, Naibo J, Jewell Joseph S et al (2020) $100 \mathrm{kHz}$ PLEET velocimetry in a Mach-6 Ludwieg tube. Opt Express 28(15):21982-21992. https://doi.org/10.1364/OE.391302
Huntley MB, Wu P, Miles RB, Smits AJ (2000) MHz rate imaging of boundary layer transition on elliptic cones at Mach 8. AIAA Pap No. https://doi.org/10.2514/6.2000-379

Jiang N, Halls BR, Stauffer HU, Danehy PM, Gord JR, Roy S (2016) Selective two-photon absorptive resonance femtosecond-laser electronic-excitation tagging velocimetry. Opt Lett. https://doi. org/10.1364/OL.41.002225

Jiang NB, Mance JG, Slipchenko MN, Felver JJ, Stauffer HU, Yi TX, Danehy PM, Roy S (2017a) Seedless velocimetry at $100 \mathrm{kHz}$ with picosecond-laser electronic-excitation tagging Opt. Lett 42(2):239-242. https://doi.org/10.1364/OL.42.000239

Kohse-Höinghaus K, Jeffries JB (2002) Applied combustion diagnostics. Taylor Francis, New York

Kulatilaka W, Gord JR, Viswanath R, Roy S (2012) Photolytic-interference-free, femtosecond two-photon fluorescence imaging of atomic hydrogen. Opt Lett 37(15):3051-3053. https://doi.org/10. 1364/OL.37.003051

Laufer G, McKenzi RL, Fletcher DG (1990) Method for measuring temperatures and densities in hypersonic wind tunnel air flows using laser-induced $\mathrm{O}_{2}$ fluorescence. Appl Opt 29(33):4873-4883. https://doi.org/10.1364/AO.29.004873

Lee WC, Lempert WR (2002) Spectrally filtered Raman/Thomson scattering using a rubidium Vapor filter. AIAA J 40(12):25042510. https://doi.org/10.2514/2.1594

Lucht RP, Roy S, Meyer TR, Gord JR (2006) Femtosecond coherent anti-stokes Raman scattering measurement of gas temperatures from frequency-spread dephasing of the Raman coherence. Appl Phys Lett 89(25):251112. https://doi.org/10.1063/1.2410237

Michael J, Edwards MR, Dogariu A, Miles RB (2011) Femtosecond laser electronic excitation tagging for quantitative velocity imaging in air. Appl Opt 50:5158. https://doi.org/10.1364/AO. 50.005158

Miles R, Limbach C, Gerakis A (2020) Filtered low angle Thomson Scattering. PDL-04 - novel approaches in diagnostics and experimental technique (ID 231) 2020 AIAA Aviation Forum, June 16, 2020

Miles R, Lempert W (1990) Two-dimensional measurement of density, velocity, and temperature of turbulent air flows from UV Rayleigh scattering. Appl Phys B 51(1):1-7

Miles RB, Lempert WR (1997) Quantitative flow visualization in unseeded flows. Annu Rev Fluid Mech 29:285-326. https://doi. org/10.1146/annurev.fluid.29.1.285

Miles Richard B (2015) Optical diagnostics for high-speed flows. Prog Aerosp Sci 72:30-36. https://doi.org/10.1016/j.paerosci. 2014.09.007

Miles Richard B, Yalin Azer P, Tang Zhen, Zaidi Sohail H, Forkey Joseph N (2001) Flow field imaging through sharp-edged atomic and molecular 'notch' filters. Meas Sci Technol 12 (4):442-451 www.iop.org/Journals/mt

Miles Richard, Dogariu Arthur, Michael James, Edwards Mathew (2018) Femtosecond laser excitation tagging anemometry US. Issued January 9, 2018

Miles Richard, Dogariu Arthur (2020) Slow light imaging spectroscopy US. Issued March 3, 2020

Miller Joseph D, Slipchenko Mikhail N, Meyer Terrence R, Stauffer Hans U, Gord James R (2010) Hybrid femtosecond/picosecond coherent anti-Stokes Raman scattering for high-speed gas-phase thermometry. Opt Lett 35(14):2430-2432. https://doi.org/10. 1364/OL.35.002430

Naibo J, Matthew W, Lempert Walter R et al (2011) MHz-rate nitric oxide planar laser-induced fluorescence imaging in a Mach 10 hypersonic wind tunnel. Appl Opt 50(4):A20-A28. https://doi. org/10.1364/AO.50.000A20

Niemi K, der Schulzvon GV, D“obele HF (2001) Absolute calibration of atomic density measurements by laser-induced fluorescence 
spectroscopy with two-photon excitation. J Phys d: Appl Phys 34(15):2330-2335

Noullez A, Wallace G, Lempert W, Miles RB, Frisch U (1997) Transverse velocity increments in turbulent flow using the RELIEF technique. J Fluid Mech 339(1997):287-307. https://doi.org/10. 1017/S0022112097005338

Palmer RR (1989) "The CARSFT computer code for calculating coherent anti-Stokes Raman spectra: user and programmer information", Sandia National Laboratories Report SAND898206. Livermore, California

Pestov D, Murawski RK, Ariunbold GO, Wang X, Zhi M, Sokolov AV, Sautenkov VA, Rostovtsev YV, Dogariu A, Huang Y, Scully M (2007) Optimizing the laser-pulse configuration for coherent Raman spectroscopy. Science 316(5822):265-268. https://doi. org/10.1126/science. 1139055

Poggie J, Erbland PJ, Smits AJ, Miles RB (2004) Quantitative visualization of compressible turbulent shear flows using condensate-enhanced Rayleigh scattering. Exp Fluids 37(3):438-454

Prince BD, Chakraborty A, Prince BM, Stauffer, (2006) Development of simultaneous frequency- and time-resolved coherent anti-Stokes Raman scattering for ultrafast detection of molecular Raman spectra. J Chem Phys 135(4):044502. https://doi.org/ $10.1063 / 1.2219439$

Rad A, Rekhy A, Dogariu A, Miles RB (2020) Slow light imaging spectroscopy (SLIS): a comparison of different filters, June 15-19, 2020 AIAA Aviation 2020 Forum. https://doi.org/10.2514/6. 2020-3242

Reese Daniel T, Naibo J, Paul D (2020) Unseeded velocimetry in nitrogen for high-pressure, cryogenic wind tunnels: Part III resonant femtosecond-laser tagging. Meas Sci Technol 31(7):075203

Reinert JD, Candler GV, Komives JR (2020) Simulations of unsteady three-dimensional hypersonic double-wedge flow experiments. AIAA J 58(9):4055-4067. https://doi.org/10.2514/1.J058789

Roy S, Miller JD, Slipchenko MN, Hsu PS, Mance JG, Meyer TR, Gord JR (2014) 100-ps-pulse-duration, 100-J burst-mode laser for kHz-MHz flow diagnostics. Opt Lett 39(22):6462-6465. https:// doi.org/10.1364/OL.39.006462

Sanchez-Gonzalez R, Bowersox RDW, North SW (2012) Simultaneous velocity and temperature measurements in gaseous flowfields using the vibrationally excited nitric oxide monitoring technique: a comprehensive study. Appl Opt 51(9):1216-1228. https://doi. org/10.1364/AO.51.001216

Settersten TB, Patterson BD, Gray JA (2006) Temperature-and speciesdependent quenching of NO A $(2) \operatorname{Sigma}(+)\left(\mathrm{v}\left({ }^{\prime}\right)=0\right)$ probed by two-photon laser-induced fluorescence using a picosecond laser. J Chem Phys 124(23):234308. https://doi.org/10.1063/1.2206783

Slipchenko MN, Meyer TR, Roy S (2021) Advances in burst-mode laser diagnostics for reacting and nonreacting flows. Proc Combust Inst 38(1):1533-1560. https://doi.org/10.1016/j.proci.2020. 07.024

Smits AJ, Lim TT (2012) Flow visualization-techniques and examples, 2nd edn. Imperial College Press, London

Smyser ME, Slipchenko MN, Meyer TR, Caswell AW, Roy S (2019) Burst-mode laser architecture for the generation of high-peak-power MHz-rate femtosecond pulses. OSA Continuum 2:3490-3498. https://doi.org/10.1364/OSAC.2.003490

Smyser ME, Braun EL, Athmanathan V, Slipchenko MN, Roy S, Meyer TR (2020) Dual-output fs/ps burst-mode laser for megahertzrate rotational coherent anti-Stokes Raman scattering. Opt Lett 45:5933-5936. https://doi.org/10.1364/OL.404984

Surzhikov ST (2018) Calculation analysis of the experimental data of HIFiRE-I using the computer code NERAT-2D. J Phys: Conf Ser 1009:012022

Taylor M, Paul D, Stephen J, Naibo J, Matthew W, Walter L, Joseph M, Terry M (2011) Stereoscopic planar laser-induced fluorescence imaging at $500 \mathrm{kHz}$. AIAA Pap. https://doi.org/10.2514/6. 2011-985

Thurow BS, Jiang N, Lempert WR, Samimy M (2005) Development of megahertz-rate planar doppler velocimetry for high-speed flows. AIAA J. https://doi.org/10.2514/1.7749

Thurow B, Jiang N, Lempert W (2013) Review of ultra-high repetition rate laser diagnostics for fluid dynamic measurements. Meas Sci Tech 24(1):012002. https://doi.org/10.1088/0957-0233/24/1/ 012002

UTA, News Release (2020), Milestone measurements in arc-jet plasma wind tunnel - News Center - The University of Texas at Arlington (uta.edu)

Wang LJ, Kuzmich A, Dogariu A (2000) Gain-assisted superluminal light propagation. Nature 406:277-279. https://doi.org/10.1038/ 35018520

Wu P, Lempert WR, Miles RB (2000) Megahertz pulse-burst laser system and visualization of shock-wave/boundary-layer interaction. AIAA J 38(4):672-679. https://doi.org/10.2514/2.1009

Wu P (2000) MHz rate pulse burst laser imaging system: development and application in the high speed diagnostics. Ph.D. Theses Princeton University

Yibin Z, Garrett M, Beresh Steven J et al (2020) Multi-line FLEET by imaging periodic masks. Opt Lett 45(14):3945-3948. https://doi. org/10.1364/OL.392779

Zaidi SH, Tang Z, Yalin AP, Barker P, Miles RB (2002) Filtered Thomson scattering in an argon Plasma. AIAA J 40(6):1087-1093. https://doi.org/10.2514/2.1756

Zhang Y, Richardson DR, Beresh SJ, Casper KM, Soehnel M, Henfling J, Spillers R (2019) Hypersonic wake measurements behind a slender cone using FLEET velocimetry. AIAA Paper. https:// doi.org/10.2514/6.2019-3381

Zimmermann M, Miles RB (1980) Hypersonic-helium flow field measurements with the resonant Doppler Velocimeter. Appl Phys Lett 37(10):885-887. https://doi.org/10.1063/1.91784

Publisher's Note Springer Nature remains neutral with regard to jurisdictional claims in published maps and institutional affiliations 